\title{
Rigidity and stability of cold dark solid universe model.
}

\author{
R.A. Battye, ${ }^{1}$ B. Carter, ${ }^{2}$ E. Chachoua,${ }^{2}$ and A. Moss $^{1}$ \\ ${ }^{1}$ Jodrell Bank Observatory, School of Physics and Astronomy, \\ University of Manchester, Macclesfield, Cheshire SK11 9DL, UK. \\ ${ }^{2}$ LuTh, Observatoire de Paris, Meudon, 92195 France.
}

(Dated: 20th May, 2005)

\begin{abstract}
The large scale dynamics of the universe appears to be dominated by a "dark energy" constituent with negative pressure to density ratio $w=P / \rho$, which could be stable if sufficiently rigid, but not if purely fluid. It was suggested by Bucher and Spergel that such a cosmological solid might be constituted by a cold (static) distribution of cosmic strings with $w=-1 / 3$, or membranes with the observationally more favoured value $w=-2 / 3$, but it was not shown that the rigidity actually would be sufficient for stability. For cases in which the defect lattice is formed from even junctions, it is found that the rigidity to density ratio will be given by $\mu / \rho=4 / 15$ in both the string and membrane cases, and it is confirmed that this is indeed sufficient for stabilisation with respect to sufficiently small perturbations.
\end{abstract}

PACS numbers: 95.35.+d, 98.80.-k

\section{INTRODUCTION}

It was pointed out by Bucher and Spergel [1] that an underlying dark energy component consisting of a frozen network of topological defects, having an approximately uniform (unclustered) density distribution $\rho$ and a strongly negative value of its pressure $P$, could account for many of the observed features of the universe on a large scale. In particular, the cosmic acceleration suggested by measurements of type Ia supernovae. It was suggested [2] that, due a symmetry breaking phase transition at a cosmological temperature of a few hundred $\mathrm{KeV}$ or less, such a constituent might be provided by a suitable distribution of membranes (domain walls), whose averaged tension would be able to provide a negative pressure, provided the positive pressure contribution from kinetic effects is not too large, i.e. provided the distribution is effectively cold enough to be treated as an approximately static "frozen" state.

For ordinary Nambu-Goto type cosmic strings, Bucher and Spergel noted [1, 2] that in a static isotropic distribution the pressure to density ratio, $w=P / \rho$ (in units with unit light speed) would be given by $w=-1 / 3$. Moreover, for ordinary Dirac type membranes (such as simple domain walls) in a cellular lattice the value would be $w=-2 / 3$, which is fairly close to what is considered [3, 4] to be observationally favoured.

The optimism of earlier [1, 2] and some more recent 3, 4, 5] discussions of such a proposal has been challenged from various points of view. One form of objection concerns conceivable observable consequences [6] derived from assumptions that are themselves open to question. However, it would seem that the most fundamental problem is the absence, so far, of any convincing suggestion as to how an effectively "hot", wiggly, disordered structure - such as would naturally emerge from a Kibble-type spontaneous symmetry breaking - could be damped in such a way as to "freeze" as an effectively "cold" lattice of straight string or flat wall segments, as is required for the pressure to become effectively negative. The positive pressure contribution from wiggles and waves will of course tend to be removed naturally by standard damping mechanisms, but the problem is that such processes [7, 8] will usually do so by removing the segments involved (so that the mean pressure to density ratio remains positive), and will not necessarily tend toward a regular lattice.

The present work does not attempt to address this basic problem of finding a plausible mechanism for setting up a cold string or membrane system of the postulated kind in the first place. Our purpose here is just to provide a better understanding of the subsequent mechanical behaviour of such a system - assuming it has already been somehow created - and in particular to investigate conditions under which such a negative pressure system can be stable.

For a purely fluid medium, negative pressure would of course entail local instability on a short timescale, as symptomised by an imaginary value for the sound speed $c_{\mathrm{S}}$ given by

$$
c_{\mathrm{S}}^{2}=\frac{\mathrm{d} P}{\mathrm{~d} \rho} .
$$

In response to this implied objection, Bucher and Spergel pointed out that a medium constituted of cosmic strings or membranes would behave like an elastic solid, rather than a perfect fluid, which can be stable even when the pressure is negative, provided the rigidity is sufficient.

The aim of the present work is to justify this claim, at least for membrane systems which contain only even junctions. However our calculation does not apply to wall systems with odd junctions, which we expect to be 


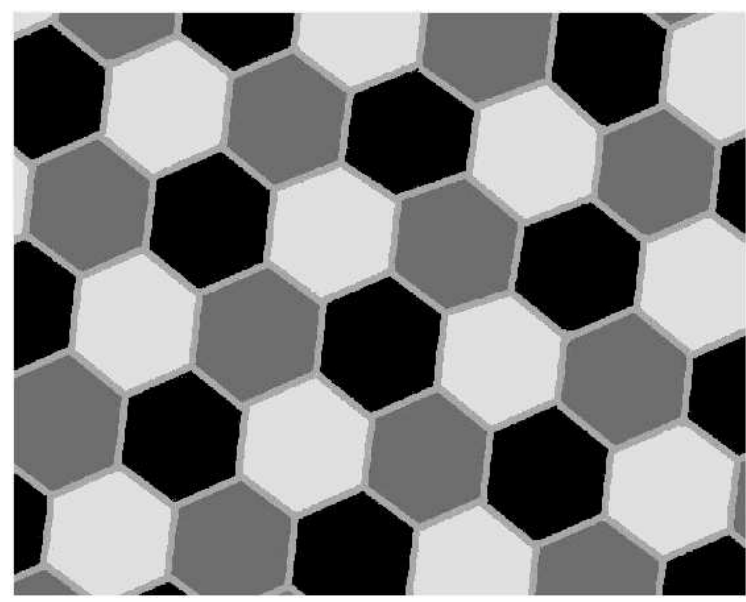

FIG. 1: Illustration of two dimensional lattice of the odd type, to which the present analysis does not apply. Each of the three colours corresponds to a different vacuum state. This hexagonal lattice is provided by a complex scalar field model of the form (2) with $M=3$.

much less rigid, so that they are likely to be, typically, too unstable for relevance as a dominant dark energy constituent. (If the walls were sufficiently massive, due to formation by symmetry breaking at very high energy, systems with odd junctions might nevertheless have been relevant to the primeval structure formation mechanism discussed in refs. 9, 10]). Our quantitative estimates will be based on the supposition that it is a sufficiently good approximation to treat the system as effectively isotropic on a macroscopic scale (meaning large compared with the mesoscopic lattice separation scale) but this simplifying condition does not seem essential for our qualitative conclusions, which we expect to remain valid even after allowance for small deviations from macroscopic anisotropy.

\section{DISTINCTION BETWEEN "EVEN" AND "ODD" TYPE SYSTEMS}

The calculation we present here will specifically be concerned with membrane systems that can be described as even-type, meaning systems where the number of walls intersecting at each (string-like) junction is even. Alternatively, if the number of walls meeting at each junction is odd, this will described as of odd-type. Mixed systems containing both odd- and even-type junctions might arise in sufficiently complicated field models, but systems of that kind have not been put forward to date and will not be considered here

Simple odd- and even-type systems can be obtained in two dimensions from complex scalar field models with a potential

$$
V\{\Phi\}=\lambda\left|\Phi^{M}-\eta^{M}\right|^{2},
$$

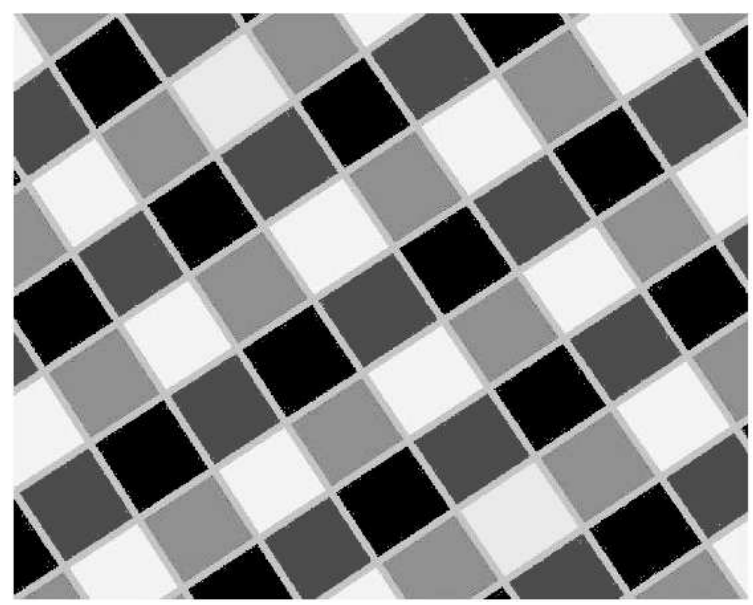

FIG. 2: Analogue of Fig. 1 with four differently coloured vacuum states obtained from (2) by setting $M=4$. This case provides a lattice of the even type to which the present analysis applies.

where $M, \lambda$ and $\eta$ are constants. There are $m$ minima of this potential at $\Phi=\eta \exp [i m / M]$ for $m=0,1, . ., M-$ 1. For $M=3$ one can construct a hexagonal lattice as illustrated in Fig. 1 which is clearly of the odd-type, whereas for $M=4$ an even lattice as illustrated in Fig. 2 is possible. We note that the stability of such systems, which can be derived from a super-potential [1]], has been investigated in ref. 12].

Our analysis will be based on the postulate that the affine displacements completely describe the modification of the equilibrium distribution at a mesoscopic level (meaning large compared with the membrane thickness, but small compared with the lattice separation scale), that is, we will assume that the local equilibrium of the system will preserved by arbitrary affine space transformations. We describe this as the affine equilibrium preservation postulate.

This postulate is applicable to lattices of the even-type, for which the flat membrane sheets cross straight through each other (as in an X-form) at an angle that can freely adjusted without affecting the condition of equilibrium. However, it can be expected to fail for odd-type systems: for example at a (Y-form) junction between 3 equivalent membrane segments in equilibrium the intersection angle must always be $2 \pi / 3$, a condition that will not be preserved by a deformation that is affine on a mesoscopic scale. In such a case, a macroscopically affine deformation would therefor entail non affine (rigidity lowering) adjustments at the mesoscopic level. The effect of a locally affine (shear wave) transformation on the two dimensional complex scalar field model with $M=4$ is illustrated in Fig. 3

More complicated, and possibly stable, systems of both odd and even type type can be obtained from perturbed $\mathrm{O}(\mathrm{N})$ models with potential energy density given by $\mathrm{N}$ 


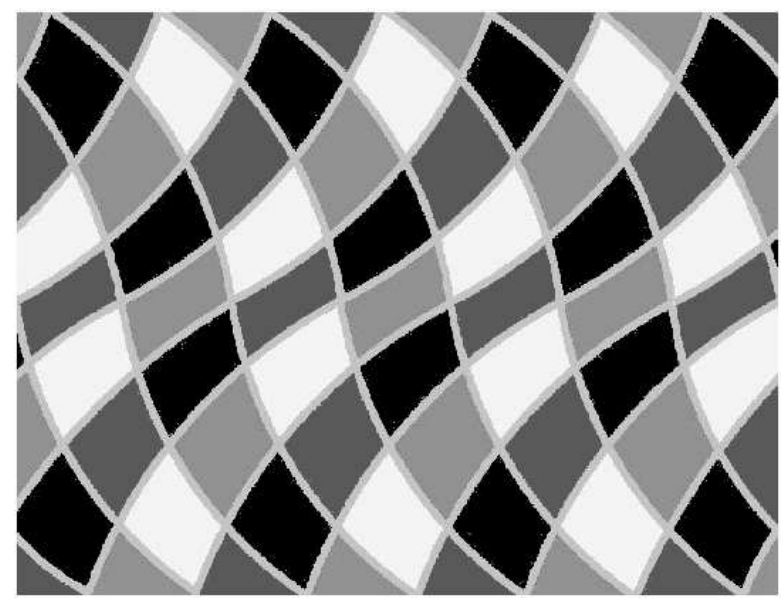

FIG. 3: The effect of a (vertically propagating) shear wave of simple mesoscopically affine type on the even type lattice illustrated in Fig 2 It is clear that the local equilibrium of each of the junctions is maintained under this locally affine deformation. This would not be possible for the hexagonal lattice illustrated in Fig. 1] for which a shear deformation could be affine only at a macroscopically averaged scale.

real scalar fields $\Phi_{i}$, and mass scales $\eta, \zeta$, in the form

$$
V\{\Phi\}=\lambda\left(\sum_{i} \Phi_{i}^{2}-\eta^{2}\right)^{2}+\mathcal{E} \sum_{i}\left(\Phi_{i}^{2}-\zeta^{2}\right)^{2},
$$

where $\lambda$ and $\mathcal{E}$ are dimensionless parameters such that $\mathcal{E}>-\lambda, \mathrm{N} \lambda>-\mathcal{E}$. The exactly $\mathrm{O}(\mathrm{N})$ symmetric model is obtained by taking $\mathcal{E}=0, \lambda>0$, while the simplest example of an even-type system is provided by the decoupled limit, $\lambda=0, \mathcal{E}>0$, for which the domain walls simply pass through each other without interaction.

Less trivial possibilities include the systems with $\zeta=0$ considered by Kubotani and collaborators [9, 10], who specifically envisaged odd-type systems - with triply intersecting ( $\mathrm{Y}$-form) junctions - obtained for $\mathrm{N}=3$ by taking $\mathcal{E}<0$. In this case there are 6 vacua which can be thought of as being at the centre of the faces of a cube. The alternative, of relevance for the present work, is the even case - with quadruple (X-form) junctions [5] between walls separating vacuum domains where $\Phi_{i}^{2}=\left(\lambda \eta^{2}+\mathcal{E} \zeta^{2}\right) /(\mathrm{N} \lambda+\mathcal{E})$ for $i=1, . ., N$ - which can be obtained (for any value of $\mathrm{N}$ ) by taking the symmetry breaking parameter to be positive, $\mathcal{E}>0$. For $\mathrm{N}=3$ there are 8 vacua at the vertices of a cube.

Field models like this engender lattices of periodic or quasi-periodic type, with walls grouped in mutually parallel families, the prototype example being that of the cubic lattice for $\mathrm{N}=3$, in which there are just 3 families arranged so as to be mutually orthogonal. The question of whether disordered (glass-type) configurations can be obtained from other plausible models remains unclear. Although its averaged stress tensor will be isotropic, the cubic $(\mathrm{N}=3)$ case (see Fig. (4) will be characterised by an elasticity tensor that distinguishes between directions

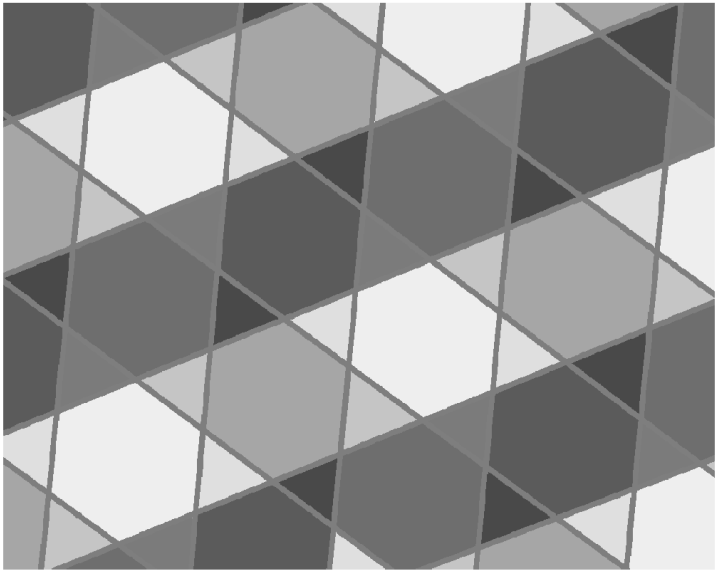

FIG. 4: Illustration of a 2 dimensional section through a frozen cubic lattice of "even" type, having (differently shaded) domains bounded by flat walls with $\mathrm{X}$ form junctions, obtained for system with eightfold vacuum provided by broken $\mathrm{O}(\mathrm{N})$ model with $\varepsilon>0$ and $\mathrm{N}=3$. Such a configuration has an averaged stress tensor that will already be exactly isotropic, but to get a very highly isotropic elasticity tensor a rather larger value of $\mathrm{N}$ would be needed.

parallel to the planes of the walls and directions that can deviate from these planes by as much as $\pi / 4$. For larger values of $\mathrm{N}$, and correspondingly larger numbers of families of mutually parallel walls, the possible deviations will get progressively smaller so that, like the stress, the ensuing elasticity properties will become highly isotropic, as postulated in the analysis below.

\section{LOCAL STABILITY CONDITION}

For a system of the even-type just described, a lattice of flat membrane states continuing straight through each other - as if without interaction - at simple crossover junctions will evidently provide a local equilibrium configuration in a flat space background. Such an equilibrium will clearly be stable at the mesoscopic scale characterised by the lattice cells, because the unbounded effectively non-interacting - membrane sheets are each individually stable with respect to local perturbations, provided their amplitude is small compared with the lattice spacing, so as to prevent distinct vacuum domains of the same type from colliding.

Assuming that - at a macroscopic scale large compared with the cell spacing - such a lattice interacts with other (relatively lightweight) constituents of the cosmic background sufficiently to form an effectively coherent medium (like a gas of point particles on scales large compared with the mean free path) one would expect that this medium would also be stable, which means having enough effective rigidity to provide real values for the relevant squared velocities of propagation. 
To verify that, for an approximately isotropic unperturbed configuration, this will indeed be so, we need the formula for the effect of rigidity on the relevant propagation velocity. This was first studied 13 when relativistic elasticity theory was originally developed for application to the solid crust of a neutron star 14], but the same formalism applies equally well here. As in the terrestrially familiar non-relativistic limit, there will be transversely polarised - shake type - modes with propagation speed $c_{\perp}$, as well as a longitudinally polarised - sound type mode with propagation speed $c_{\|}$.

It was shown [13] that the latter would exceed the ordinary fluid sound speed value (11) by an amount proportional to the rigidity modulus $\mu$ according to the formula

$$
c_{\|}^{2}=c_{\mathrm{S}}^{2}+\frac{4}{3} c_{\perp}^{2}
$$

in which the squared shake mode speed is given by

$$
c_{\perp}^{2}=\frac{\mu}{\rho+P} .
$$

For stabilisation of the medium, that is to ensure that $c_{\|}^{2}$ will be positive even if $c_{\mathrm{S}}^{2}$ is not, the necessary and sufficient condition is therefore

$$
\mu>-\frac{3}{4} \beta
$$

where $\beta$ is the bulk modulus, as defined in the relativistic case [13, 14] by the formula

$$
\beta=(\rho+P) \frac{\mathrm{d} P}{\mathrm{~d} \rho} .
$$

This reduces to the familiar form $\beta=\rho \mathrm{d} P / \mathrm{d} \rho$ in the nonrelativistic limit $|P| \ll \rho$. The requirement (6) simplifies to $\mu>-3 \gamma P / 4$ in the case of a polytropic equation of state, defined in terms of a conserved number density $n$ and constants $\gamma, \kappa$, and $m$ by $\rho=\kappa n^{\gamma}+m n$ which implies $P=(\gamma-1) \kappa n^{\gamma}$.

The models considered here correspond to the zeromass limit, $m=0$, in this polytropic class, and the bulk modulus will be given by

$$
\beta=w \gamma \rho, \quad w=\gamma-1
$$

Since we have $\gamma=2 / 3$ in the string case, and $\gamma=1 / 3$ in the membrane case, it follows that the bulk modulus will have a negative, and thus destabilising, value that will be given by the same formula,

$$
\beta=-\frac{2}{9} \rho
$$

in both string and membrane cases. It can be seen that this result will still be valid for a non-polytropic mixture with $\rho=\kappa_{1} n^{1 / 3}+\kappa_{2} n^{2 / 3}$. Therefore, either separately and also for a mixed system of strings and membranes, the stability criterion (6) will reduce to a requirement of the same simple form,

$$
\frac{\mu}{\rho}>\frac{1}{6} .
$$

\section{EVALUATION OF RIGIDITY}

The new result provided by the present work for the even-type lattices described above is the explicit evaluation of the rigidity $\mu$, and the confirmation that the ratio $\mu / \rho$ - which turns out to be the same for strings as for membranes - actually will satisfy the stability condition (10).

The required rigidity, or shear, modulus $\mu$ can be defined, for an initially isotropic state, by expressing the static - quadratic order - change in the mass-energy density $\rho$ due to an infinitesimal volume conserving space displacement as [13, 14, 15]

$$
\Delta \rho=\mu e_{i j} e^{i j},
$$

where $e_{i j}$ is the infinitesimal strain tensor. For an affine displacement given in Cartesian coordinates by $x^{i} \mapsto \tilde{x}^{i}=x^{i}+\varepsilon^{i}{ }_{j} x^{j}$, it is defined as the symmetric part of the deformation matrix: $e_{i j}=\left(\varepsilon_{i j}+\varepsilon_{j i}\right) / 2$.

In order to avoid an extra energy variation contribution due to the pressure, we impose the restriction that the displacement must conserve volume. This requires that the Jacobean determinant $\left|\partial \tilde{x}_{i} / \partial x_{j}\right|$ should be unity which implies that the trace $e_{i}^{i}$, like the variation $\Delta \rho$, will vanish to linear - though not higher - order in $\varepsilon$. An obvious way to do this is to take the symmetric (zero curl) pure shear deformation given in terms of coordinates $x^{1}=x, x^{2}=y, x^{3}=z$ by $\tilde{x}=(1+\varepsilon) x, \tilde{y}=y$, $\tilde{z}=z /(1+\varepsilon)$, which when substituted in (11) gives

$$
\Delta \rho=2 \mu \varepsilon^{2},
$$

to second order in $\varepsilon$. The value of $\mu$ can be read out from this when the variation $\Delta \rho$ has been worked out.

However, the required value of $\mu$ can be obtained in a computationally more convenient way by considering the effect on $\rho$ of the asymmetric (non-zero curl) simple shear deformation given by

$$
\tilde{x}=x+2 \varepsilon z, \quad \tilde{y}=y, \quad \tilde{z}=z,
$$

which is of the kind produced dynamically by a transverse mode with propagation speed in the $z$ direction, given by (5). It can be checked that a simple shear deformation such as this will provide a second order expression of the same form (12) to the one obtained for the pure shear deformation considered above.

To evaluate the density variation $\Delta \rho$ produced - as the left hand side of (12) - by the action of the simple volume conserving transformation (13) on a distribution 
of randomly oriented strings, let us consider the case of a sample string segment from the origin to a point with coordinates given by the components, $\{x, y, 1\}$ say, of a vector $\vec{\ell}$, which will be mapped to a vector $\vec{\ell}+\Delta \vec{\ell}$ with coordinates $\{x+2 \varepsilon, y, 1\}$. The length $\ell=\left(x^{2}+y^{2}+1\right)^{1 / 2}$ of the segment will undergo a fractional change given, to quadratic order in $\varepsilon$, by

$$
\frac{\Delta \ell}{\ell}=\frac{2 x \varepsilon}{\ell^{2}}+\frac{2\left(\ell^{2}-x^{2}\right) \varepsilon^{2}}{\ell^{4}} .
$$

Since the mass-energy of a static Nambu Goto string segment is obtained simply by multiplying its tension, $T$ say, the formula (14) immediately provides the fractional change in the contribution to the mass density $\rho$ from strings with the chosen direction, as given in polar coordinates by $x=\tan \theta \cos \phi, y=\tan \theta \sin \phi$.

Since the model is based on the postulate that the strings have a random isotropic distribution, their net effect can be computed by just taking the spherical average of (14) over the polar coordinate values $\theta, \phi$, which gives a formula in which the first order part cancels out, leaving just the quadratic term,

$$
\frac{\Delta \rho}{\rho}=\left\langle\frac{\Delta \ell}{\ell}\right\rangle=\frac{8}{15} \varepsilon^{2} .
$$

To evaluate the effect of the same simple shear deformation (13) on an isotropic distribution of plane membranes, consider a parallelogram shaped sample segment spanned by two vectors, $\vec{\ell}_{1}$, with components $\left\{x_{1}, 1,0\right\}$, which will not change, and $\vec{\ell}_{2}$, with components $\left\{x_{2}, 0,1\right\}$, which will be deformed to $\left\{\left(x_{2}+\right.\right.$ $2 \varepsilon), 0,1\}$. The membrane analogue of the string formula (15) for the effect on the density of an isotropic distribution is

$$
\frac{\Delta \rho}{\rho}=\left\langle\frac{\Delta A}{A}\right\rangle,
$$

where $A$ is the sample area. This is given by the magnitude of the vector product $\vec{A}=\vec{\ell}_{1} \times \vec{\ell}_{2}$, with components $\left\{1, x_{1},-x_{2}\right\}$ in the direction normal to the membrane. The deformation (13) will map this areacalibrated normal vector to $\vec{A}+\Delta \vec{A}$, with components $\left\{1, x_{1},-\left(x_{2}+2 \varepsilon\right)\right\}$. Since this transformation differs from that of the string segment considered above only by a permutation of the roles of the $x$ and $z$ axes, the final result, after spherical averaging, will be the same.

For the macroscopically isotropic and mesoscopically even-type systems under consideration, this reasoning provides a theorem to the effect that - like the bulk modulus $\beta$ as given by (9) - the rigidity modulus $\mu$ of the isotropic cosmic membrane distribution will be identical to that of an isotropic cosmic string distribution with the same density $\rho$. Thus, by comparing (15) with (12), it can be seen that the value of the rigidity is

$$
\mu=\frac{4}{15} \rho
$$

not only in the string case, but also in the membrane case and for a mixed system of strings and membranes.

\section{CONCLUSIONS}

It is evident that the value given by (17) does indeed satisfy the stability condition (10). This confirms the intrinsic coherence of the solid universe model subject to the availability of an underlying field theory of a suitable kind, meaning one providing a membrane system of eventype, for example, (3) with $\mathcal{E}>0$. However, it remains unclear how such a system could have come into existence in the first place.

In a solid of this kind, as a consequence of (4) and (5), we obtain real values for the speeds of longitudinal as well as transverse modes. For the latter, (15) with (17) gives $c_{\perp}^{2}=4 / 15 \gamma$, so the (transverse) shake wave speed will be given by

$$
\gamma=2 / 3 \Rightarrow c_{\perp}^{2}=2 / 5, \quad \gamma=1 / 3 \Rightarrow c_{\perp}^{2}=4 / 5,
$$

for the string and membrane cases respectively. The corresponding formula obtained from (4) for the longitudinal wave speed will take the form

$$
c_{\|}^{2}=w+\frac{16}{45 \gamma},
$$

which will indeed be consistent with the stability criterion (6), since it clearly provides a real-valued propagation speed for any positive value of the polytropic index,

$$
\gamma=w+1
$$

In particular it can be seen that the longitudinal propagation speed will be given by

$$
w=-1 / 3 \Rightarrow c_{\|}^{2}=1 / 5, \quad w=-2 / 3 \Rightarrow c_{\|}^{2}=2 / 5,
$$

in the string and membrane cases respectively, the latter being the one of greatest cosmological interest.

The present analysis is based on the cold limit in which thermal [16] or other kinetic excitations of the world sheets are neglected. If significant, such effects would tend [17] to reduce the effective tension, giving slower perturbation speeds and also making it harder to explain the apparent 3] cosmic acceleration. Such effects would also tend to undermine the stability of the system, which is valid (as shown above) only for perturbation amplitudes that are small compared with the relevant lattice separation scale. The fundamental difficulty with this kind of scenario is the problem of finding a natural mechanism for getting rid of such excitation effects so as to actually obtain a frozen lattice of the required kind.

Assuming that the membrane domination scenario can be achieved, and that it could be obtained by some spontaneous symmetry breaking process, a first attempt was 
made 2] at evaluating the energy scale, $\eta$ say, characterising the tension, $T \approx \eta^{3}$, of the required membranes and the cosmological temperature, $\Theta \approx \eta$, of their presumed formation. It relied on the assumption that the relevant correlation lengthscale at the epoch of formation would be the maximum permitted by causality. In a recent reconsideration of this issue by one of the present authors [5] it has be shown that, although it would be more realistic to suppose the correlation length to be very much smaller than the causal limit, nevertheless the effect of the appropriate correction on the estimate for $\eta$ is relatively moderate: the likely range is merely reduced from the order of a hundred $\mathrm{KeV}$ to a few $\mathrm{KeV}$. This does not change the qualitatively important consequence that the transition should have occurred at a relatively recent cosmological era, after pair annihilation and Big-Bang Nucleosynthesis, though before the epoch of recombination when the universe became transparent. The corollary is that the present lattice separation scale of the membrane lattice will be comparable [2, 5], at most, with interstellar rather than intergalactic separation lengthscales. Thus, on the presumption that the relevant fields interact very weakly with ordinary particles such as electrons, and particularly photons, so that the walls will not be directly visible, Bucher and Spergel's provisional conclusion that their scenario poses no hierarchy problem remains valid, in the sense that the range of conceivable values for $\eta$ overlaps with the mass range for already known elementary particles.

Despite this attractive feature, the plausibility of such a scenario is undermined by the absence, as remarked above, of any mechanism for getting from a randomly excited initial state to a regular frozen membrane lattice of the required kind, as exemplified by the case depicted in Figure 1. In view of the positive results of our study, this issue warrants further investigation.

The authors wish to thank Martin Bucher for instructive conversations.

[1] M. Bucher, D.N. Spergel, "Is the dark matter a solid", Phys. Rev. D60 (1999) 043505. astro-ph/9812022
[2] R.A. Battye, M. Bucher, D.N. Spergel, "Domain wall dominated universes". astro-ph/9908047

[3] L. Conversi, A. Melchiorri, L. Mersini, J. Silk, "Are domain walls ruled out?", Astropart. Phys. 21 (2004) 443449. astro-ph/0402529

[4] R.A. Battye, A. Moss, "Constraints on the solid dark universe model", to appear in JCAP. astro-ph/0503033

[5] B. Carter, "Frozen rigging model of the energy dominated universe", contribution to Micro and Macro Structures of Space Time (Peyresq, June 2004) ed. E Verdaguer, to be published in Int. J. Theor. Phys. hep-ph/0412397

[6] G.D. Starkman, D.B. Stojkovic, "How Frustrated Strings Would Pull the Black Holes from the Centers of Galaxies" Phys. Rev. D63 (2001) 045008. astro-ph/0010563

[7] U.L. Pen and D.N. Spergel, 'Cosmology in a string dominated universe", Ap. J. 491 (1997) L67-L71.

[8] P.N. McGraw "Evolution of a non-Abelian cosmic string network", Phys. Rev. D57 (1998) 33173339. astro-ph/9706182

[9] H. Ishahara, H. Kubotani, Y. Nambu, "The domain wall network of explicitly broken $0(\mathrm{~N})$ model", Prog. Theor. Phys. 87 (1992) 387-400.

[10] M. Den, K. Yamashita, H. Kubotani, "Testing the domain wall dominated scenario for the evolution of the large scale structure of the universe" , Prog. Theor. Phys. 103 (2000) 559-571.

[11] G.W. Gibbons and P.K. Townsend, "A Bogomolyni equation for intersecting domain walls", Phys. Rev. Lett. 83 (1999) 1727-1730.

[12] P. Saffin, "Tiling with almost-BPS junctions", Phys. Rev. Lett. 83 (1999) 4249-4252.

[13] B. Carter, "Speed of sound in a high pressure general relativistic solid", Phys. Rev. D7 (1973) 1590-1593.

[14] B. Carter, "Interaction of Gravitational Waves with an Elastic Solid Medium", in Gravitational Radiation (Proc. 1982 Les Houches Summer School), ed N. Deruelle \& T. Piran, (North Holland, Amsterdam, 1983) 455-464. gr-qc/0102113

[15] L.D. Landau, E.M. Lifshitz, Theory of Elasticity, (Pergamon, London, 1959) 10-12.

[16] B. Carter, "Cold, Warm, and Composite (cool) Cosmic String models", Nuclear Physics B412 (1994) 345-371. hep-ph/0210263

[17] B. Carter, "Transonic elastic model for wiggly GotoNambu strings", Phys. Rev. Letters 74 (1995) 3098-3101. hep-th/9411231 